Many such screw 'deformations' occur in bending chromium platelets of this kind.

Chicago Development Corporation,

R. S. DEAN

F. X. MCCAwLeY

Riverdale, Maryland.

${ }^{1}$ Dean, R. S., and McCawley, F. X., Nature, 180, 435 (1957).

\section{A Highly Sensitive Chemical Dosimeter for lonizing Radiation}

An aqueous dosimeter capable of measuring radiation doses of less than 100 rads would be an extremely useful tool in the field of radiobiology. Although doses of a few rads can be accurately measured using the resorcinol-stabilized tetrachlorethylene-dye systems ${ }^{\mathbf{1}}$, such systems where the radiation-induced reaction proceeds by a chain mechanism exhibit certain undesirable characteristics. An alternative to the radiation-induced chain reaction involves the measurement of extremely small amounts of an irradiation product by means of a sensitive analytical technique. Some idea of the extreme sensitivity required is obtained from the fact that a dose of 100 rads will produce approximately $10^{-7}$ moles of product per litre of solution, assuming a reasonable production-rate of unity for $G_{\text {prod. }}$ (that is to say, 1 molecule of product formed per $100 \mathrm{eV}$. absorbed). An analytical method capable of accurate measurement at such high dilution is spectrophotofluorometry. The production of salicylic acid (which fluoresces in aqueous solution) in irradiated aqueous solutions of benzoic acid seemed to merit investigation, especially because this reaction had been proposed $^{2}$ as suitable for chemical dosimetry.

During irradiation a mixture of the three isomeric hydroxybenzoic acids is produced in the ratio of $5: 2: 10$ for the $o: m: p$ isomers respectively ${ }^{3}$. However, at $400 \mathrm{~m} \mu$ (exciting wave-length $290 \mathrm{~m} \mu$ ) the fluorescent intensity of the $m$ and $p$ isomers is small compared with that of salicylic acid and can be neglected. Diphenyl has also been identi-

fied as a radiation product, but it does not fluoresce at $400 \mathrm{~m} \mu$ if excited at $290 \mathrm{~m} \mu$. The fluorescent intensity of salicylic acid solutions is a linear function of concentration in the range $10^{-7}-10^{-8} \mathrm{M}$, and is independent of acidity from $p H$ 5-11. Since the reproducibility of the measurements was only about \pm 10 per cent, presumably because of the fluctuations in the intensity of the light source of the AmincoBowman spectrophotofluorometer, each experimental run was calibrated separately in the following manner. The fluorescent intensities of two standard salicylic acid solutions were measured immediately before and immediately after measurements were carried out on a series of irradiated solutions, and the salicylic acid concentrations in the latter solutions were computed using the average value of the 'fluorescence coefficient' :

Fluorescent coefficient $=$

fluorescent intensity of standard [salicylic acid]
All dose-rates were determined using the ferrous sulphate $-0.8 \mathrm{~N}$ sulphuric acid dosimeter ${ }^{4}$. Corrections for the decreased energy absorption in water were made as described previously ${ }^{5}$.

From Fig. 1 it is seen that the concentration of salicylic acid in irradiated $10^{-4} M$ calcium benzoate solutions $(p H 5)$ increases in a linear fashion with dose of $X$ - and $\gamma$-radiation over the range $0-100$ rads, and is independent of dose-rate over the range 4-50 rads/min. For a given dose the fluorescent intensity is almost identical for cobalt- 60 and crsium$137 \gamma$-rays. The rates of salicylic acid production expressed as $G_{\text {sal. }}$ were computed to be : Incident

$\begin{array}{ccccc}\text { Incident } & & & \\ \text { radiation } & \mathrm{Co}^{60} & \mathrm{Cs}^{137} & 300-\mathrm{kV} & 100-\mathrm{kV} . \\ \text { Effective } & \gamma \text {-rays } & \gamma \text {-rays } & \text { neak } \mathrm{X} \text {-rays } & \text { peakX-rays } \\ \text { energy } & & & & \end{array}$

energy

(MeV.) $\begin{array}{cccc}1 \cdot 25 & 0.66 & 0.16 & 0.05\end{array}$

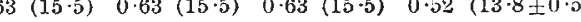

The values in parentheses refer to the values of the oxidation yield of the ferrous sulphate dosimeter used in the computation of $G$ sal. 